\title{
Regional Adrenal Gland Pheochromocytoma
}

National Cancer Institute

\section{Source}

National Cancer Institute. Regional Adrenal Gland Pheochromocytoma. NCI Thesaurus.

Code C8045.

A pheochromocytoma that has not spread to other regions. 